\title{
Microarray analysis of the molecular mechanisms associated with age and body mass index in human meniscal injury
}

\author{
PEIYAN HUANG, JUN GU, JUNGUO WU, LEI GENG, YANG HONG, SIQUN WANG and MINGHAI WANG \\ Department of Orthopedic Surgery, Shanghai Fifth People's Hospital \\ Affiliated to Fudan University, Shanghai 200240, P.R. China
}

Received February 4, 2018; Accepted October 4, 2018

DOI: $10.3892 / \mathrm{mmr} .2018 .9685$

\begin{abstract}
The aim of the present study was to identify genes and functional pathways associated with meniscal injuries affected by age or body mass index (BMI) using microarray analysis. The GSE45233 gene expression dataset with 12 injured meniscus samples associated with age and BMI and GSE66635 dataset with 12 injured and 12 normal meniscus samples were downloaded from the Gene Expression Omnibus database. Differentially expressed genes (DEGs) were identified based on age or BMI in GSE45233. DEGs between injured and normal meniscus samples in GSE66635 were also identified. Common DEGs between GSE45233 and GSE66635 were identified as feature genes associated with age or BMI, followed by protein-protein interaction (PPI) network and functional pathway enrichment analyses for the feature genes. Finally, the GSE51588 genome-wide expression profile was then downloaded from the GEO database to validate the results. A total of 1,328 DEGs were identified. Of these, 28 age-associated and 20 BMI-associated meniscal injury genes were obtained. B-cell lymphoma-2 (Bcl-2) and matrix metalloproteinase-14 were identified as hub genes in the PPI networks. Functional pathway enrichment analysis revealed that vascular endothelial growth factor $\mathrm{A}$ (VEGFA), transferrin (TF) and Bcl-2 were involved in the hypoxia-inducible factor 1 signaling pathway. TF was involved in the mineral absorption function pathway associated with BMI. Additionally, TF and VEGFA were identified to be overlapping candidate genes of GSE45233 and GSE66635, and DEGs in GSE51588. Therefore, VEGFA, TF, and Bcl-2 may be important genes for human meniscal injuries. Additional evaluations of these results are required.
\end{abstract}

Correspondence to: Dr Minghai Wang, Department of Orthopedic Surgery, Shanghai Fifth People's Hospital Affiliated to Fudan University, 128 Ruili Road, Minhang, Shanghai 200240, P.R. China E-mail:wwmmhai2@163.com

Key words: meniscal injury, body mass index, age, differentially expressed genes, protein-protein interaction network, functional enrichment analysis

\section{Introduction}

Meniscal injury is a common disorder that may cause pain, swelling or mechanical symptoms in patients (1). As articular cartilages of the knee joint, menisci are crucial for normal function of the knee joint and protection of the articular surfaces (2). Articular cartilages, as specialized connective tissues of diarthrodial joints, are affected by overall fitness levels $(3,4)$.

Previously, the effect of factors including aging and obesity on articular cartilages has been widely studied. With aging, changes occur in articular cartilages, including degenerative changes in the morphology, loss of cartilage matrix proteins, cleavage of type II collagen, worsening of mechanical properties and dysregulated expression of associated genes (4-7). An inverse correlation between cartilage thickness within the knee joint and age has been demonstrated (8). The mechanisms of obesity affecting articular cartilages are complex, and include biomechanical effects via increased loading and meta-inflammation (9-14). Abnormalities in the articular cartilage of the knee are also associated with obesity, as revealed by the investigation of obese adolescent patients suffering from knee pain (15).

Details of the gene expression profile in human meniscal injury have been described previously (16-18). Expression of vascular endothelial growth factor A (VEGFA), tumor necrosis factor- $\alpha$ and matrix metalloproteinases (MMPs) were demonstrated to be varied by age in patients with meniscal tears $(16,19)$. Neuronal apoptosis-inhibitory protein, apoptosis inhibitor of macrophage, carbohydrate sulfotransferase 15 and MMP28 were suggested to be associated with obesity (17). However, gene expression changes associated with injuries and aging or obesity in the human meniscus have not been fully investigated.

Microarray analysis of gene expression changes and functional pathways may be useful to improve understanding of the associations between aging or obesity and meniscal injuries. In previous studies, GSE45233 microarray data was used to study variation in gene expression signatures in human injured meniscus with age or body mass index (BMI) and the degree of chondrosis in the knee $(16,17)$. GSE66635 microarray data was used to compare transcriptome signatures between the meniscus and articular cartilage from knees undergoing arthroscopic partial meniscectomy (18). In the present study, the GSE45233 and GSE66635 datasets were downloaded and analyzed to identify genes and functional pathways associated with age or BMI in human injured meniscus. Differentially 
expressed genes (DEGs) were identified and feature genes were obtained by comparing the DEGs from the 2 datasets. Protein-protein interaction (PPI) networks of these feature genes were constructed to obtain hub genes. Functional pathway enrichment analysis for feature genes was also performed to identify key pathways in the development of age or BMI-associated meniscal injuries. Furthermore, the GSE51588 genome-wide expression profile was downloaded from the Gene Expression Omnibus (GEO) database to validate the results. The results concerning gene expression changes in human injured meniscus may provide an improved understanding of the associations between meniscal injuries and aging or obesity.

\section{Materials and methods}

Affymetrix microarray data. The gene expression profile datasets GSE66635, deposited by Rai et al (18), and GSE45233, deposited by Rai et al $(16,17)$, were downloaded from the GEO (www.ncbi.nlm.nih.gov/geo/) database. GSE66635 and GSE45233 were based on the GPL16686 Human Gene 2.0 ST Array (Affymetrix Inc.; Thermo Fisher Scientific, Inc., Waltham, MA, USA) and GPL10558 HumanHT-12 V4.0 expression Beadchip (Illumina, Inc., San Diego, CA, USA) platforms, respectively. A total of 24 samples were available in the GSE66635 dataset, including 12 injured meniscus and 12 normal articular cartilages from patients undergoing partial meniscectomy. The second microarray profile, GSE45233, included 12 isolated injured meniscus tissues from patients undergoing arthroscopic partial meniscectomy.

Data preprocessing and feature genes screening. The probe-level data in the CEL files were converted to expression measures, and idle probes were deleted. When multiple probes corresponded to the same gene, the expression values of those probes were averaged. The 2 datasets were aggregated according to gene names, followed by background correction and quartile data normalization by the robust multiarray average algorithm in R language (20).

Based on the GSE66635 gene expression profile data, DEGs in injured menisci from patients undergoing partial meniscectomy were identified using the Linear Models for Microarray Data (Limma) package (21) in R language compared with normal articular cartilage. The cut-off values were $\mathrm{P}<0.05$ and fold change $(\mathrm{FC})>1.5$.

Samples of GSE45233 were grouped by age and BMI according to World Health Organization standards (lean, BMI $<18.5$; normal, BMI 18.5-25; obese, BMI > $25 \mathrm{~kg} / \mathrm{m}^{2}$ ) (22). The age group included 6 samples of patients $>40$ years of age (old group) and 6 samples of patients $<40$ years of age (young group). DEGs associated with age were then identified in the old group using the Limma package and compared with the young group.

The BMI group contained 8 samples of lean patients (lean group) and 4 samples of obese patients (obese group); no patients were in the normal category according to this dataset. DEGs associated with age were identified in the obese group using the Limma package and compared with the lean group.

Common DEGs between GSE66635 and the age group in GSE45233 were defined as 'feature' genes associated with age-associated meniscal injuries. Similarly, common DEGs identified from GSE66635 and the BMI group in GSE45233 were BMI-associated feature genes.

Integrative analysis of feature gene expression levels. A total of 9 samples of patients $>40$ years of age and 3 samples of patients $<40$ years of age were selected from the injured meniscus samples in GSE66635. Combining these samples with those in the age group from GSE45233, a new age group with 15 samples of patients $>40$ years of age and 9 samples of patients $<40$ years of age was obtained. Similarly, 8 obese samples from GSE66635 were selected and combined with those in the BMI group from GSE45233, obtaining a new BMI group with 8 lean samples and 12 obese samples. The significances of average expression level differences for feature genes extracted from the two new groups were analyzed by t-test using the Limma package (23). Heat maps were generated using Pheatmap (24) in R language, to exhibit the results of bidirectional hierarchical clustering based on the expression value of the feature genes.

Classification of the sample classification model. Support vector machines (SVM) (25) are a useful technique for two-group classification problems. To verify the identifiability and classifiability of the feature genes extracted from the two new groups, the SVM classifier model was performed based on the expression value of these feature genes. SVMs were initially optimized using the DEGs in the training set and the feature genes were examined in the test set to separate the samples in the new age and BMI groups.

PPI network construction. The Human Protein Reference Database (HPRD; www.hprd.org/) (26) is a protein database that contains information on human protein functions, including PPIs, post-translational modifications, and enzyme-substrate and disease associations. PPI networks for age-associated feature genes and BMI-associated feature genes were respectively constructed by mapping the two groups of feature genes to the PPI pairs downloaded from HPRD, which were then visualized using Cytoscape (27). Finally, hub genes with the highest node degree in the PPI networks were identified using CytoNCA (28) plugin (network without weight) in Cytoscape.

Pathway enrichment analysis. The Kyoto Encyclopedia of Genes and Genomes (KEGG) pathway database (www. genome.jp/kegg/pathway.html) (29) is used for the systematic analysis of gene functions and associated genomic information with higher order functional information. The feature genes were examined using the KEGG pathway database to identify functional pathways. The enrichment process was performed by the Fisher algorithm using KOBAS2.0 (30) as follows:

$$
\mathrm{P}=1-\sum_{i=0}^{x-1} \frac{\left(\begin{array}{l}
\mathrm{M} \\
\mathrm{i}
\end{array}\right)\left(\begin{array}{l}
\mathrm{N}-\mathrm{M} \\
\mathrm{K}-\mathrm{i}
\end{array}\right)}{\left(\begin{array}{l}
\mathrm{N} \\
\mathrm{K}
\end{array}\right)}
$$

where $\mathrm{N}$ is the number of total genes in the genome, $\mathrm{M}$ is the number of genes in the pathway and $\mathrm{K}$ is the number of DEGs. The Fisher score indicates the probability of $\mathrm{X}$ functional pathway genes among K DEGs. 
Data validation. To validate the results, the GSE51588 genome-wide expression profile was downloaded from the GEO database. This dataset was obtained from 40 subchondral bone tissue samples from patients with osteoarthritis and 10 samples from normal subjects based on the GPL13497 Agilent-026652 Whole Human Genome Microarray 4x44 K v2 platform. The original data in TXT format were preprocessed using the Limma package (version 3.30.13; www. bioconductor.org/packages/2.9/bioc/html/limma.html) (21). The preprocessing methods included background correction and data normalization. DEGs were identified using the Bayes method in Limma with a threshold $\mathrm{P}<0.05$.

The overlapping DEGs between GSE66635 and GSE45233 were selected as candidate genes, which were then compared with the DEGs obtained from GSE51588.

\section{Results}

Screening of candidate feature genes. Following data normalization, genes exceeding the difference threshold $(\mathrm{P}<0.05$ and FC >1.5) were screened. A total of 364 DEGs were identified in injured meniscus samples compared with normal meniscus samples in GSE66635, among which 135 DEGs were downregulated and 229 DEGs were upregulated. A total of 424DEGs were screened in the old group compared with the young group in GSE45233, including 222 downregulated genes and 202 upregulated genes. In the obese group, compared with the lean group, 540 genes were differentially expressed, including 340 downregulated and 200 upregulated genes. Comparison of the DEGs in the GSE66635 dataset with the DEGs of the age group in GSE45233 identified 28 age-associated meniscal injury feature genes (Table I). Of these, 15 feature genes were downregulated, which included S100 calcium-binding protein A1 (S100A1) and BARX homeobox 2, and 13 feature genes were upregulated, which included cluster of differentiation 36 and endomucin (EMCN). A total of 20 common genes between the GSE45233 dataset and the BMI group in GSE45233 were identified as BMI-associated meniscal injury feature genes (Table I). Of these, 11 feature genes were downregulated, which included EMCN and furry, and 9 feature genes were upregulated, which included IGF binding protein 1 and S100 calcium-binding protein A8 (S100A8).

Integrative analysis of expression levels of feature genes. Following integration of the GSE66635 and GSE45233 datasets, a new age-associated meniscal injury group including 24 samples and a new BMI-associated meniscal injury group including 20 samples were obtained. Intra-group differences of the 28 age-associated meniscal injury feature genes and $20 \mathrm{BMI}$ associated meniscal injury feature genes were examined by t-test. For the age group, the average expression levels of the young group were significantly increased compared with those of the old group $(\mathrm{P}=0.021)$; for the BMI group, the average expression levels of the lean group were increased compared with those of the obese group, but the difference was not significant ( $\mathrm{P}=0.3663$; Fig. 1A). Heat maps (Fig. 1B and $\mathrm{C}$ ) of bidirectional hierarchical clustering indicated that the samples within the age group were completely separated into the young and old groups, while 1 obese sample (obese-12) in the BMI group was assigned to the lean group.
Table I. Feature genes in age and body mass index groups.

\begin{tabular}{|c|c|c|}
\hline Gene & P-value & $\log _{2} \mathrm{FC}$ \\
\hline S100A1 & 0.0004 & -5.64 \\
\hline BARX2 & 0.0096 & -2.95 \\
\hline IGFBP1 & 0.0341 & -2.46 \\
\hline HAPLN1 & 0.0031 & -2.21 \\
\hline DIXDC1 & 0.0123 & -2.01 \\
\hline FGFBP2 & 0.0381 & -1.92 \\
\hline FBLN7 & 0.0076 & -1.81 \\
\hline VEGFA & 0.0173 & -1.66 \\
\hline GREM1 & 0.0171 & -1.56 \\
\hline $\mathrm{TF}$ & 0.0281 & -1.40 \\
\hline BCL2 & 0.0345 & -1.30 \\
\hline DCHS1 & 0.0020 & -1.29 \\
\hline SMOC1 & 0.0042 & -1.23 \\
\hline CAPN6 & 0.0167 & -1.20 \\
\hline PTGES & 0.0352 & -1.08 \\
\hline VIT & 0.0459 & 1.00 \\
\hline LYZ & 0.0258 & 1.23 \\
\hline FGL2 & 0.0033 & 1.24 \\
\hline CCNB1 & 0.0030 & 1.31 \\
\hline PBK & 0.0043 & 1.39 \\
\hline BST2 & 0.0102 & 1.46 \\
\hline CTSS & 0.0222 & 1.48 \\
\hline HTRA4 & 0.0068 & 1.51 \\
\hline DDHD1 & 0.0093 & 1.55 \\
\hline TSPAN7 & 0.0007 & 1.57 \\
\hline CALCRL & 0.0198 & 2.61 \\
\hline EMCN & 0.0171 & 6.40 \\
\hline CD36 & 0.0010 & 9.33 \\
\hline
\end{tabular}

B, Body mass index group

\begin{tabular}{lcc}
\hline Gene & P-value & $\log _{2} \mathrm{FC}$ \\
\hline EMCN & 0.0169 & -6.40 \\
FRY & 0.0353 & -4.20 \\
EDNRA & 0.0084 & -3.18 \\
MFAP2 & 0.0304 & -2.26 \\
CXCL12 & 0.0113 & -2.19 \\
ABHD2 & 0.0010 & -1.86 \\
EYA4 & 0.0339 & -1.79 \\
ARHGAP11B & 0.0145 & -1.57 \\
MKL2 & 0.0260 & -1.41 \\
MMP14 & 0.0398 & -1.26 \\
ISLR & 0.0121 & -1.18 \\
DIXDC1 & 0.0150 & 1.03 \\
BTC & 0.0212 & 1.14 \\
RCAN1 & 0.0191 & 1.34 \\
TF & 0.0325 & 1.45 \\
CST6 & 0.0397 & 1.48 \\
RAD51AP2 & 0.0007 & 1.58 \\
KAL1 & 0.0098 & 2.40 \\
S100A8 & 0.0042 & 2.74 \\
IGFBP1 & 0.0157 & 2.95 \\
\hline
\end{tabular}

FC, fold change. 

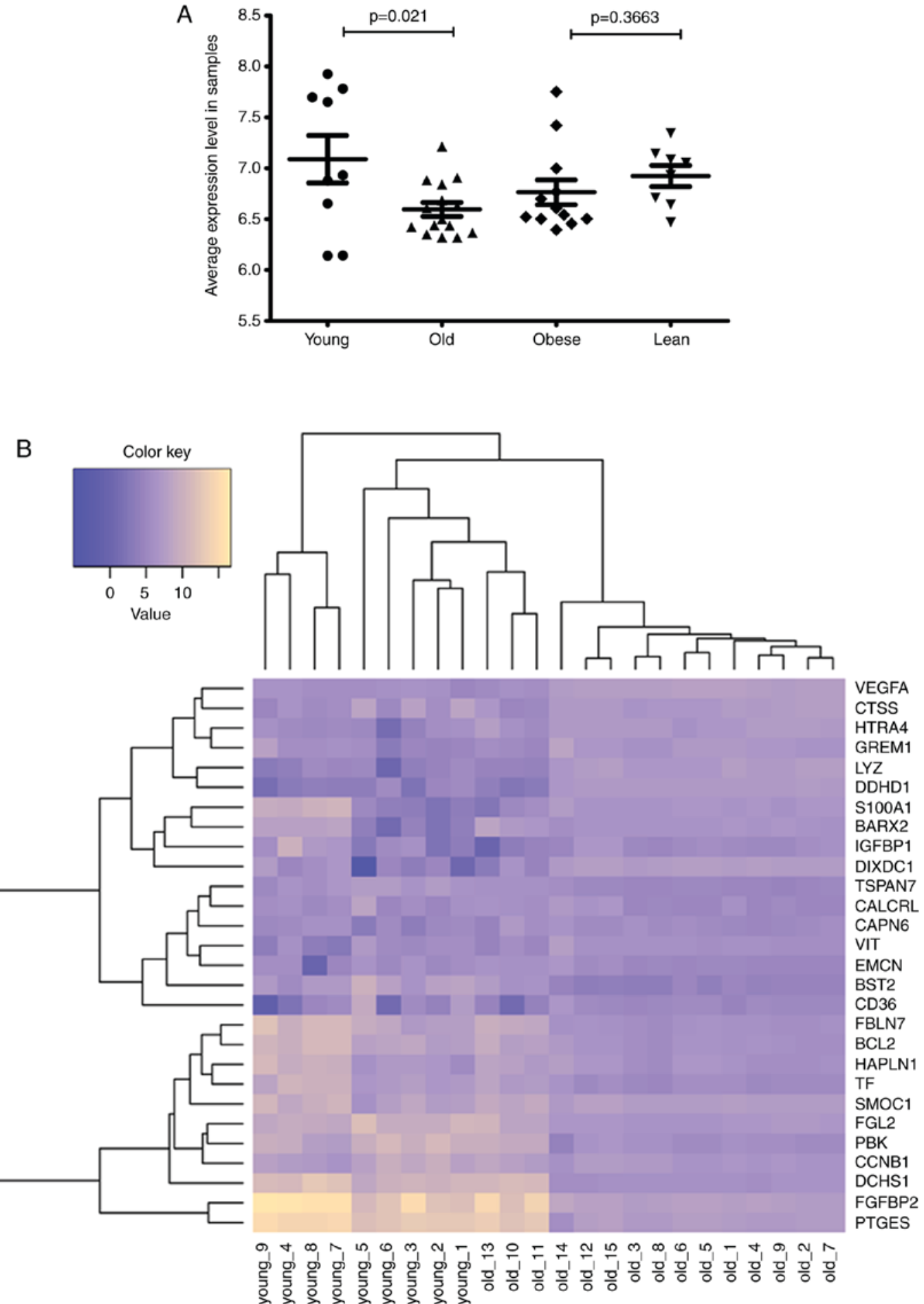

Figure 1. Comparison of the average expression levels between groups of feature genes. (A) A t-test of the average expression level of feature genes was performed. (B) Heat map of bidirectional hierarchical clustering for feature genes in the age group.

Classification of SVM classifier model. To observe the classifiability of the feature genes from the age and BMI classifications, the SVM classifier model was utilized. Samples in the age group were divided into two groups, but 1 old sample was included with the young samples (Fig. 2A). The samples in the BMI group were completely divided into lean and obese groups (Fig. 2B).

PPI network analysis. Analysis of the HPRD database identified 215 unique PPI pairs for the age group and 90 unique PPI pairs for the BMI group. The PPI network for age-associated feature genes contained 222 nodes, including 18 feature genes (Fig. 3A). The PPI network for BMI-associated feature genes contained 102 nodes, including 15 feature genes (Fig. 3B). Network topology analysis indicated that the constructed PPI networks obeyed scale-free network attributes (Fig. 4) and the node degree of the network followed the distribution, obtaining $\mathrm{y}=64.6 * \mathrm{x}^{-2.57}$ for the age group and $\mathrm{y}=47.82 * \mathrm{x}^{-2.46}$ for the BMI group, where $\mathrm{x}$ represents node degree. The top 5 nodes with the higher node degree of the 


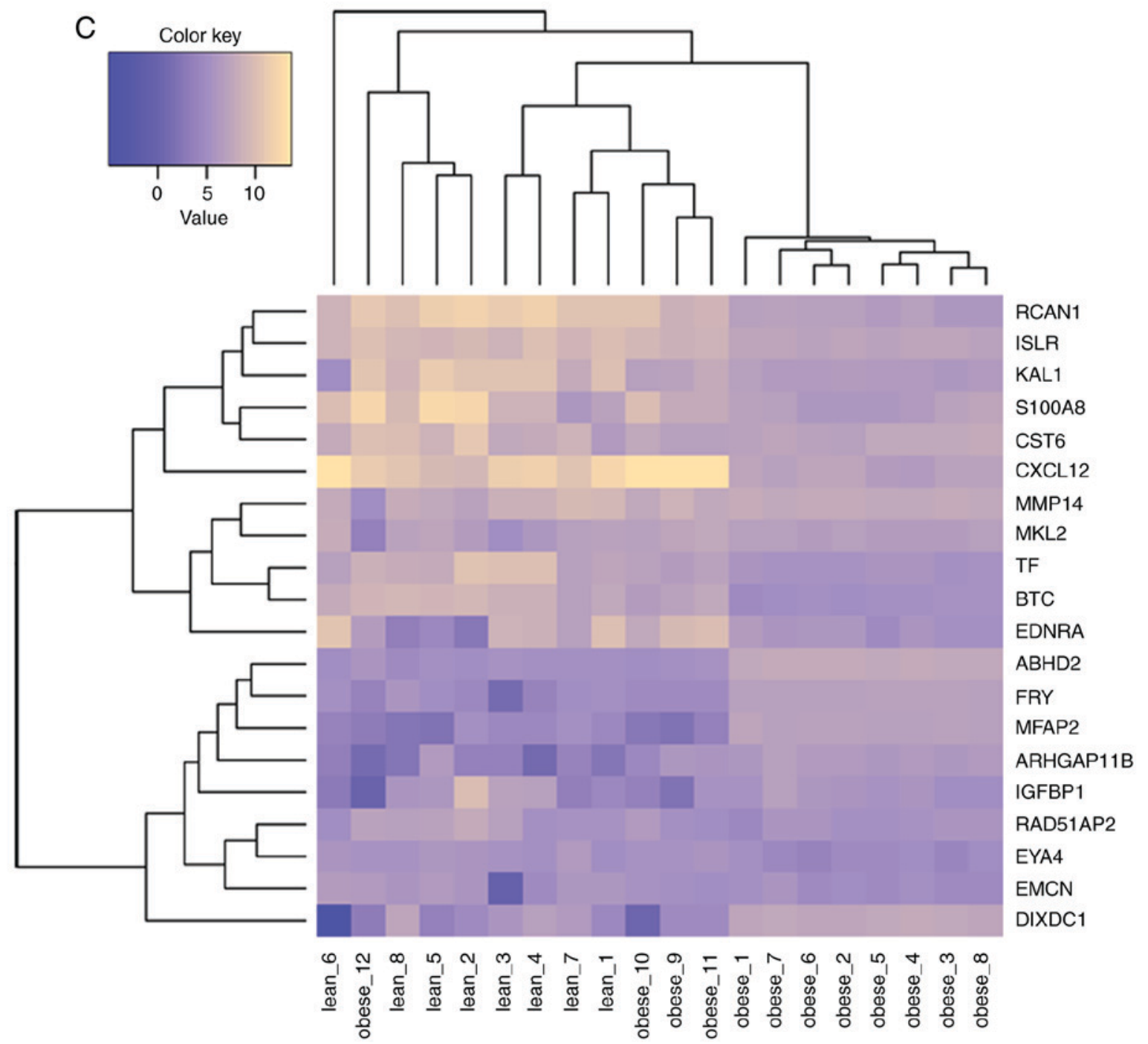

Figure 1. Continued. Comparison of the average expression levels between groups of feature genes. (C) Heat map of bidirectional hierarchical clustering for feature genes in the body mass index group.
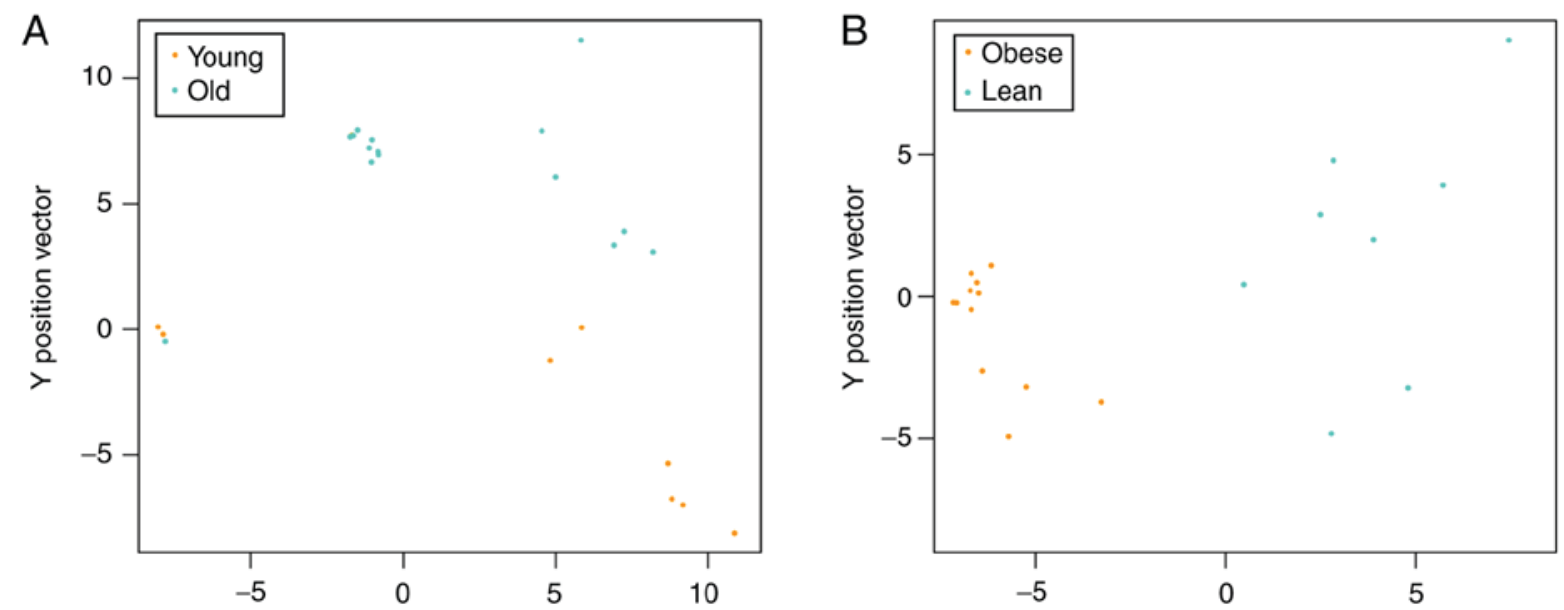

Figure 2. Scatter plots of support vector machines classification of the samples. (A) Age and (B) body mass index groups are presented. The orange color represents the young and obese samples. The green color represents old and lean samples. The x-axis and y-axis represent the sample position vector coordinates according to the classifier.

age group were B-cell lymphoma-2 (Bcl-2), CyclinB1, S100A1, VEGFA and Transferrin (TF) (Table II). The top 5 nodes of the
BMI group were MMP14, S100A8, TF, chemokine (C-X-C motif) ligand 12 and endothelin receptor type A (Table II). 


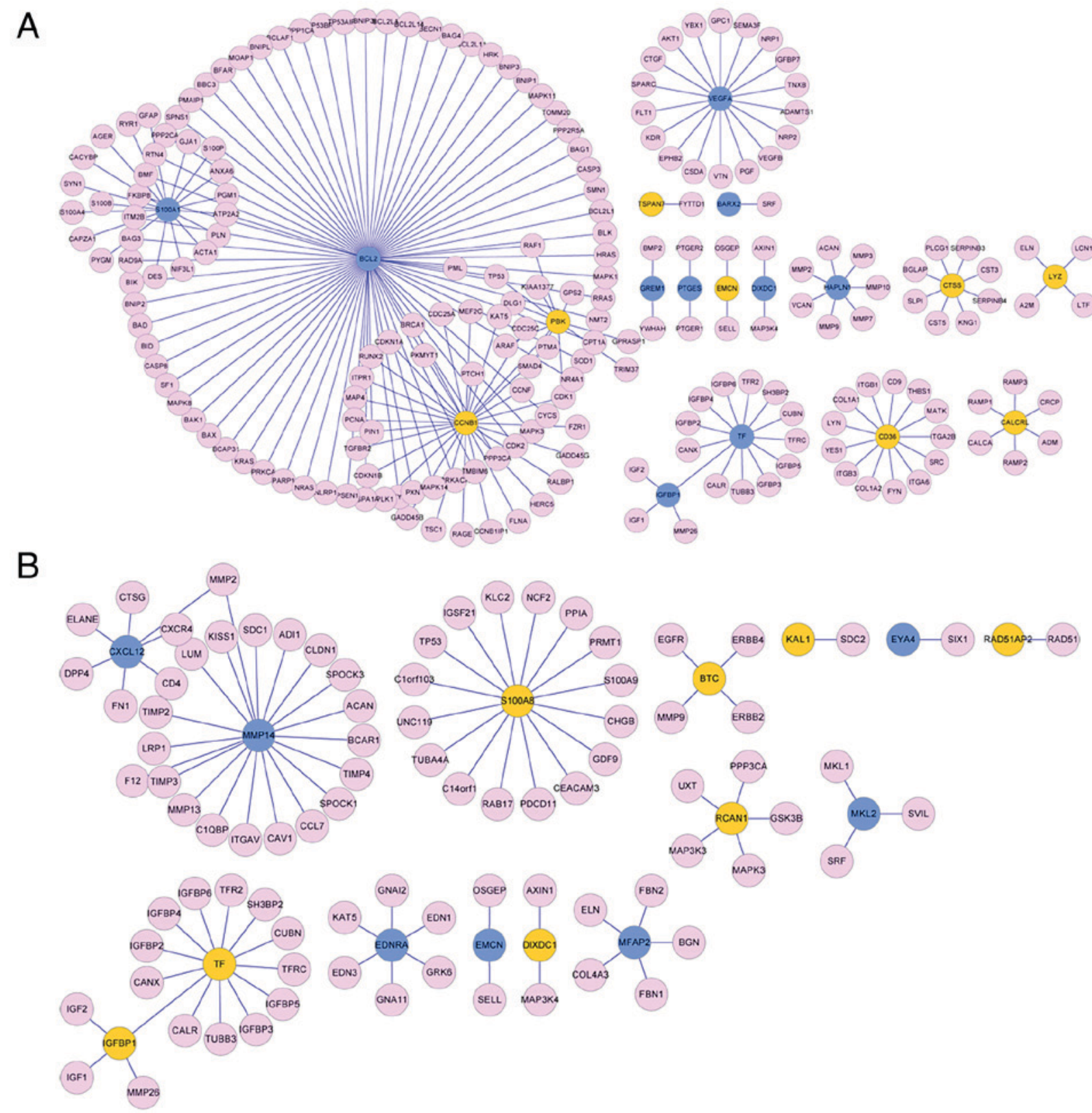

Figure 3. PPI networks of feature genes and network topology analysis. (A) PPI network constructed based on the feature genes in the age group. (B) PPI network constructed based on the feature genes in the BMI group. The yellow color represents upregulated genes and the blue color represents downregulated genes. The young group serves as the control in the age group, and the lean group serves as the control in the BMI group. The pink color represents the remaining interactional genes, with the exception of the feature genes. PPI, protein-protein interaction; BMI, body mass index.

Pathway enrichment analysis. KEGG pathway enrichment analyses for the top 10 feature genes were performed. Feature genes associated with age-associated meniscal injury were significantly enriched in the hypoxia-inducible factor 1 (HIF-1) signaling pathway, including VEGFA, TF, and Bcl-2 (Table III). Feature genes associated with BMI-associated meniscal injury were significantly associated with the mineral absorption function pathway, in which TF was enriched.

Data validation. From GSE51588, a total of 3,403 DEGs (1,853 upregulated and 1,550 downregulated) were identified (data not shown). Following comparison of GSE66635 and GSE45233, a total of 28 and 20 common DEGs (candidate genes) associated with age and BMI, respectively, were identified. Finally, 11 overlapping genes were obtained between candidate genes and DEGs in GSE51588 (Table IV). These included TF and VEGFA.

\section{Discussion}

Meniscal injury is a common disease caused by athletic events and activities in daily life. Patient age and BMI are associated meniscal injuries $(17,18)$. An understanding of the molecular mechanism of the associations between meniscal injuries and age or BMI is important. In the present study, 2 datasets (GSE45233 and GSE66635) associated with meniscal injury were downloaded from the GEO database and analyzed. Feature genes associated with age-associated meniscal injury, including VEGFA, TF and Bcl-2 were involved in the HIF-1 signaling pathway. In addition, the feature gene TF was also associated 
A

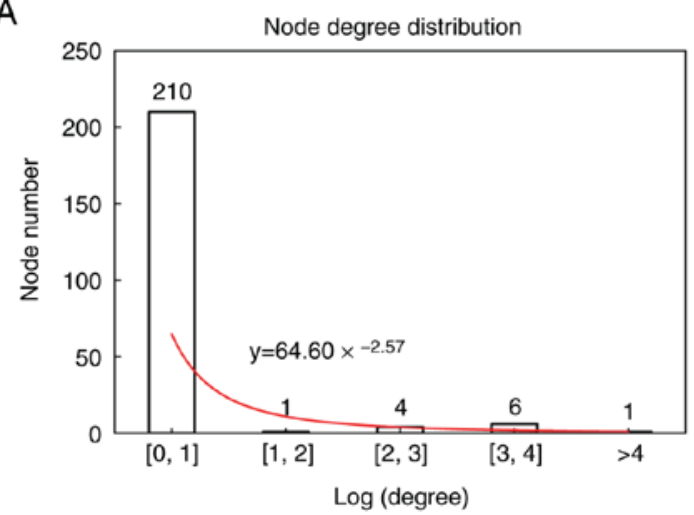

B

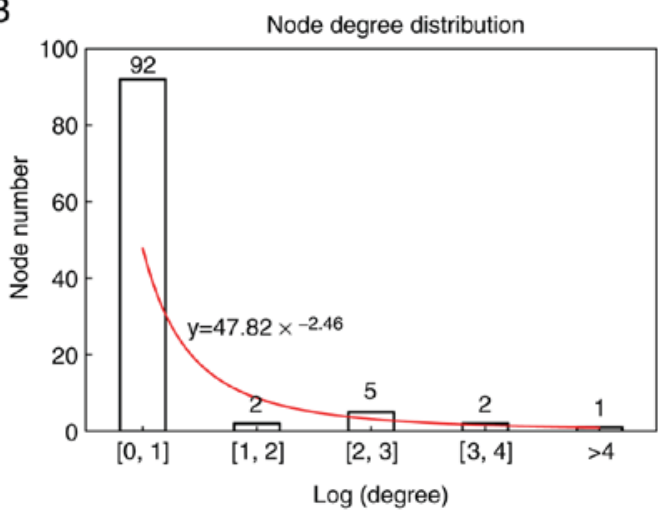

Figure 4. Node degree distribution of the feature genes. (A) Node degree distribution of the age group network. (B) Node degree distribution of the body mass index group network.

Table II. List of top 10 highest degree nodes of the age and body mass index groups.

\begin{tabular}{lcrc} 
A, Age group & & & \\
\hline Gene & P-value & $\log _{2} \mathrm{FC}$ & Degree \\
\hline BCL2 & 0.0345 & -1.30 & 74 \\
CCNB1 & 0.0030 & 1.31 & 30 \\
S100A1 & 0.0004 & -5.64 & 18 \\
VEGFA & 0.0173 & -1.66 & 18 \\
TF & 0.0281 & -1.40 & 14 \\
CD36 & 0.0010 & 9.33 & 13 \\
PBK & 0.0043 & 1.39 & 12 \\
CTSS & 0.0222 & 1.48 & 8 \\
HAPLN1 & 0.0031 & -2.21 & 7 \\
CALCRL & 0.0198 & 2.61 & 6 \\
\hline
\end{tabular}

$\mathrm{B}$, Body mass index group

\begin{tabular}{lcrc}
\hline Gene & P-value & $\log _{2} \mathrm{FC}$ & Degree \\
\hline MMP14 & 0.0398 & -1.26 & 20 \\
S100A8 & 0.0042 & 2.74 & 16 \\
TF & 0.0325 & 1.45 & 14 \\
CXCL12 & 0.0113 & -2.19 & 7 \\
EDNRA & 0.0084 & -3.18 & 6 \\
IGFBP1 & 0.0157 & 2.95 & 5 \\
MFAP2 & 0.0304 & -2.26 & 5 \\
RCAN1 & 0.0191 & 1.34 & 5 \\
BTC & 0.0212 & 1.14 & 4 \\
MKL2 & 0.0260 & -1.41 & 3 \\
\hline
\end{tabular}

with BMI-associated meniscal injury and was significantly enriched in the mineral absorption function pathway.

Bcl-2 is a regulator of apoptosis from the B-cell lymphoma-2 family, and its function is to inhibit cell death, rather than promote proliferation (18,31-33). Overexpression of $\mathrm{Bcl}-2$ in mouse bones suppresses apoptosis of bone cells (34) and suppression of Bcl-2 increases apoptosis in leukemic cells (35). Meniscal injuries are directly associated with the development of osteoarthritis (36). Emerging evidence indicates that apoptosis serves an important role in osteoarthritis pathology (37). Iwata et al (38) demonstrated that the levels of apoptosis in chondrocytes in mice consuming a high-fat diet was increased. The present study identified that Bcl-2 was downregulated in the injured meniscus samples compared with the normal meniscus, meaning that apoptosis may be increased in injured menisci tissues, which was in accordance with data from Iwata et al (38). The expression of Bcl-2 was downregulated in the old group compared with the young group, in accordance with a previous study (16). Bcl-2 was the hub gene with a node degree of 74 in the PPI network for the feature genes associated with age in the injured meniscus. All these data imply that aging may accelerate apoptosis of the injured menisci of older patients.

VEGFA is an important angiogenetic protein with a selective mitogenic effect on vascular endothelial cells (39). As a vascular endothelial growth factor, VEGFA was demonstrated to be necessary for the survival of chondrocytes during skeletal development (40) and as a regulator of osteoblast differentiation during bone development (41). VEGFA may increase the osteogenic healing capacity by promoting osteogenic and endothelial differentiation (42). A previous study revealed that VEGF levels were increased following the creation of meniscal lesions in rabbits (43). Chen et al (39) recently identified that VEGFA was downregulated in osteoarthritis chondrocytes compared with normal chondrocytes. In the present study, the expression of VEGFA was downregulated in the injured meniscus compared with the normal meniscus tissues, which is consistent with previous data that the healing capacity of menisci tissue may be damaged in injured meniscus, as the angiogenesis function of VEGFA is be inhibited in the downregulated condition $(44,45)$. Furthermore, the expression of VEGFA was downregulated in the old group compared with the young group, consistent with a previous study (16). Therefore, the healing capacity for injured meniscus of older patients may be not as efficient compared with younger patients.

In the present study, TF was identified as a meniscal injury feature gene associated with age and BMI. TF is an iron transport protein, which delivers iron from absorption centers and 
Table III. Pathways associated with feature genes in the age and BMI groups.

\begin{tabular}{lllll}
\hline Group & \multicolumn{1}{c}{ Pathway } & ID & P-value & Genes \\
\hline Age & HIF-1 signaling pathway & hsa04066 & 0.002013 & VEGFA, TF, BCL2 \\
BMI & Mineral absorption & hsa04978 & 0.043813 & TF \\
\hline
\end{tabular}

HIF-1, hypoxia-inducible factor 1; VEGFA, vascular endothelial growth factor A; TF, transferrin; BCL2, B-cell lymphoma 2; BMI, body mass index.

Table IV. Overlapping genes between candidate genes and differentially expressed genes in GSE51588.

\begin{tabular}{|c|c|c|c|c|c|}
\hline \multirow[b]{2}{*}{ Gene name } & \multicolumn{3}{|c|}{ Candidate genes } & \multicolumn{2}{|c|}{$\begin{array}{l}\text { Differentially expressed genes } \\
\text { in GSE51588 }\end{array}$} \\
\hline & P-value & $\log \mathrm{FC}$ & Type & P-value & $\log \mathrm{FC}$ \\
\hline $\mathrm{TF}$ & $2.81 \times 10^{-2}$ & -1.40 & Age & $3.20 \times 10^{-3}$ & -0.56 \\
\hline VEGFA & $1.73 \times 10^{-2}$ & -1.66 & Age & $7.95 \times 10^{-3}$ & -0.62 \\
\hline BST2 & $1.02 \times 10^{-2}$ & 1.46 & Age & $6.25 \times 10^{-3}$ & 0.64 \\
\hline EMCN & $1.71 \times 10^{-2}$ & 6.40 & Age & $1.23 \times 10^{-5}$ & 0.65 \\
\hline VIT & $4.59 \times 10^{-2}$ & 1.00 & Age & $4.17 \times 10^{-5}$ & 0.70 \\
\hline ABHD2 & $9.91 \times 10^{-4}$ & -1.86 & BMI & $2.09 \times 10^{-2}$ & -0.56 \\
\hline ARHGAP11B & $1.45 \times 10^{-2}$ & -1.57 & BMI & $1.19 \times 10^{-2}$ & -0.72 \\
\hline BTC & $2.12 \times 10^{-2}$ & 1.14 & BMI & $4.12 \times 10^{-3}$ & 1.13 \\
\hline DIXDC1 & $1.50 \times 10^{-2}$ & 1.03 & BMI & $2.27 \times 10^{-7}$ & 0.63 \\
\hline KAL1 & $9.84 \times 10^{-3}$ & 2.40 & BMI & $1.39 \times 10^{-3}$ & 0.95 \\
\hline RAD51AP2 & $6.60 \times 10^{-4}$ & 1.58 & BMI & $4.94 \times 10^{-2}$ & 0.37 \\
\hline
\end{tabular}

BMI, body mass index.

storage sites to all tissues as it circulates in the plasma (46). TF serves a major role in angiogenesis during endochondral bone formation and is produced by hypertrophic chondrocytes (47). Hypertrophy and neovascularization are specific signs of healing of injured menisci (48), through which TF may be produced and transported. Based on these data, the upregulation of TF in the injured meniscus indicates that the healing of the injured meniscus may have occurred, and that TF may have a role in the healing progress. However, this hypothesis requires experimental verification. TF was identified to be downregulated in the old group compared with the young group in the present study, which is consistent with previous data (16), indicating that recovery of the injured meniscus in old patients may be slower. Patient age has a detrimental effect on the healing potential of the injured meniscus (48). The reason why the expression of TF was upregulated in the obese group compared with the lean group is not clear, as a tendency for lower TF saturation in obesity has been suggested (49). Additional investigation is required to verify the association between TF and healing of injured meniscus.

One of the key results of the present study was that the feature genes in the age group were enriched in the HIF-1 signaling pathway and the feature genes in the BMI group were enriched in the mineral absorption function pathway. HIF-1 is a transcriptional activator that targets genes encoding proteins that increase $\mathrm{O}_{2}$ delivery and mediate adaptive responses to $\mathrm{O}_{2}$ deprivation (50,51). HIF-1 $\alpha$ may protect articular cartilage by promoting the chondrocyte phenotype, maintaining chondrocyte viability and supporting metabolic adaptation to a hypoxic environment (52). Articular cartilage is a hypoxic tissue, in which HIF-1 is essential for survival, growth, energy generation and matrix synthesis of chondrocytes $(33,44)$. HIF-1 is the major regulator of VEGFA in the context of angiogenesis $(53,54)$. VEGFA may promote angiogenesis $(44,45)$, which is an indication of the healing process in injured menisci (48). Expression of oxygen-regulated TF is positively mediated by HIF-1 (55), which occurs in the hypoxic meniscal tissues. In addition, Bcl-2 may promote HIF-1-mediated VEGF expression under hypoxia by increasing the expression level of HIF-1 (56). In the present study, VEGFA and Bcl-2 were downregulated in the injured meniscus compared with the normal meniscus tissue. This indicates that the HIF-1 signaling pathway was inhibited. These results indicate that apoptosis may be increased, and angiogenesis may be decreased in meniscal injury tissues. The expression of VEGFA, TF and Bcl-2 was downregulated in the old group compared with the young group, which may explain the longer time generally required for older patients to recover from meniscal injuries compared with younger patients (57). However, TF was upregulated in the injured meniscus, which was in contrast to the data that TF is positively mediated by 
HIF-1 under hypoxic condition (55). This may be a result of co-regulation by the HIF-1 signaling pathway and the mineral absorption function pathway. An additional contradiction is that TF was upregulated in the obese group and, contrarily, TF saturation in obesity is usually decreased (49). Experimental verification of these contradictions is important for additional understanding of the mechanisms of meniscal injuries.

There are certain limitations in the present study. The key genes identified were not validated in clinical or in vivo studies, although data validation was performed. Additionally, the sample size was small. Therefore, future clinical and in vivo studies will be performed to validate the results of the present study, and to investigate the underlying molecular mechanisms of the effects of aging and obesity on meniscal injuries.

Aging may affect the development of meniscal injuries through the HIF-1 signaling pathway, in which VEGFA, TF, and Bcl-2 are involved. Obesity may affect the mineral absorption function pathway in injured menisci by regulating the expression of TF. However, there remains a lack of experimental evidence to confirm the hypotheses in the present study, and the molecular mechanisms of the effects of aging and obesity on these pathways remain unclear. In addition, why and how TF is involved in the HIF-1 signaling pathway and the mineral absorption function pathway requires additional study.

\section{Acknowledgements}

Not applicable.

\section{Funding}

The present study was funded by the Minhang District Natural Science Project (grant no. 2017MH77).

\section{Availability of data and materials}

The analyzed data sets generated during the study are available from the corresponding author on reasonable request.

\section{Authors' contributions}

$\mathrm{PH}$ contributed to data collection, analysis and interpretation, obtained funding and wrote the manuscript. SW and $\mathrm{YH}$ contributed to the study design. LG performed the experiments and contributed to the data collection. JG performed data analysis and interpretation. JW conducted statistical analysis. MW contributed to data collection and interpretation. All authors read and approved the final manuscript.

\section{Ethics approval and consent to participate}

Not applicable.

\section{Patient consent for publication}

Not applicable.

\section{Competing interests}

The authors declare that they have no competing interests.

\section{References}

1. Greis PE, Bardana DD, Holmstrom MC and Burks RT: Meniscal injury: I. Basic science and evaluation. J Am Acad Orthop Surg 10: 168-176, 2002.

2. Noble J and Turner PG: The function, pathology, and surgery of the meniscus. Clin Orthop Relat Res: 62-68, 1986.

3. Anandacoomarasamy A, Leibman S, Smith G, Caterson I, Giuffre B, Fransen M, Sambrook PN and March L: Weight loss in obese people has structure-modifying effects on knee articular cartilage. Ann Rheum Dis 71: 26-32, 2012.

4. Musumeci G, Szychlinska MA and Mobasheri A: Age-related degeneration of articular cartilage in the pathogenesis of osteoarthritis: Molecular markers of senescent chondrocytes. Histol Histopathol 30: 1-12, 2015.

5. Armstrong CG and Mow VC: Variations in the intrinsic mechanical properties of human articular cartilage with age, degeneration and water content. J Bone Joint Surg Am 64: 88-94, 1982.

6. Buckwalter JA, Roughley PJ and Rosenberg LC: Age-related changes in cartilage proteoglycans: Quantitative electron microscopic studies. Microsc Res Tech 28: 398-408, 1994.

7. Bank RA, Bayliss MT, Lafeber FP, Maroudas A and Tekoppele JM: Ageing and zonal variation in post-translational modification of collagen in normal human articular cartilage. The age-related increase in non-enzymatic glycation affects biomechanical properties of cartilage. Biochem J 330: 345-351, 1998.

8. Dalla PL, Cova M and Pozzi-Mucelli RS: MRI appearance of the articular cartilage in the knee according to age. J Belge Radiol 80: 17-20, 1997.

9. Jones DG: Articular cartilage degeneration: Etiologic association with obesity. Ochsner J 9: 137-139, 2009.

10. Sowers MR and Karvonen-Gutierrez CA: The evolving role of obesity in knee osteoarthritis. Curr Opin Rheumatol 22: 533-537, 2010.

11. Mezhov V, Ciccutini FM, Hanna FS, Brennan SL, Wang YY, Urquhart DM and Wluka AE: Does obesity affect knee cartilage? A systematic review of magnetic resonance imaging data. Obes Rev 15: 143-157, 2014.

12. Blazek K, Favre J, Asay J, Erharthledik J and Andriacchi T: Age and obesity alter the relationship between femoral articular cartilage thickness and ambulatory loads in individuals without osteoarthritis. J Orthop Res 32: 394-402, 2014

13. Travascio F, Eltoukhy M, Cami S and Asfour S: Altered mechano-chemical environment in hip articular cartilage: Effect of obesity. Biomech Model Mechanobiol 13: 1-15, 2014.

14. Denning WM, Winward JG, Pardo MB, Hopkins JT and Seeley MK: Body weight independently affects articular cartilage catabolism. J Sports Sci Med 14: 290-296, 2015.

15. Widhalm HK, Marlovits S, Welsch GH, Dirisamer A, Neuhold A, van Griensven M, Seemann R, Vécsei V and Widhalm K: Obesity-related juvenile form of cartilage lesions: A new affliction in the knees of morbidly obese children and adolescents. Eur Radiol 22: 672-681, 2012.

16. Rai MF, Patra D, Sandell LJ and Brophy RH: Transcriptome analysis of injured human meniscus reveals a distinct phenotype of meniscus degeneration with aging. Arthritis Rheum 65: 2090-2101, 2013.

17. Rai MF, Patra D, Sandell LJ and Brophy RH: Relationship of gene expression in the injured human meniscus to body mass index: A biologic connection between obesity and osteoarthritis. Arthritis Rheumatol 66: 2152-2164, 2014.

18. Rai MF, Sandell LJ, Zhang B, Wright RW and Brophy RH: RNA microarray analysis of macroscopically normal articular cartilage from knees undergoing partial medial meniscectomy: Potential prediction of the risk for developing osteoarthritis. PLoS One 11: e0155373, 2016.

19. Brophy RH, Rai MF, Zhang Z, Torgomyan A and Sandell LJ: Molecular analysis of age and sex-related gene expression in meniscal tears with and without a concomitant anterior cruciate ligament tear. J Bone Joint Surg Am 94: 385-393, 2012.

20. Irizarry RA, Hobbs B, Collin F, Beazer-Barclay YD, Antonellis KJ, Scherf U and Speed TP: Exploration, normalization, and summaries of high density oligonucleotide array probe level data. Biostatistics 4: 249-264, 2003.

21. Smyth GK: Limma: Linear models for microarray data. In: Bioinformatics and computational biology solutions using $\mathrm{R}$ and bioconductor. Gentleman R, Carey VJ, Huber W, Irizarry RA and Dudoit S (eds.) Springer New York, NY, pp397-420, 2005. 
22. Appiah CA: World Health Organisation. Global Database on Body Mass Index. World Health Organisation: Geneva 2006, 2014.

23. Ritchie ME, Phipson B, Wu D, Hu Y, Law CW, Shi W and Smyth GK: limma powers differential expression analyses for RNA-sequencing and microarray studies. Nucleic Acids Res 43: e47, 2015.

24. Kolde R: Pheatmap: Pretty Heatmaps, 2015.

25. Cortes C and Vapnik V: Support-vector networks. Mach Learn 20: 273-297, 1995.

26. Keshava Prasad TS, Goel R, Kandasamy K, Keerthikumar S, Kumar S, Mathivanan S, Telikicherla D, Raju R, Shafreen B, Venugopal A, et al: Human protein reference database-2009 update. Nucleic Acids Res 37: D767-D772, 2009.

27. Shannon P, Markiel A, Ozier O, Baliga NS, Wang JT, Ramage D, Amin N, Schwikowski B and Ideker T: Cytoscape: A software environment for integrated models of biomolecular interaction networks. Genome Res 13: 2498-2504, 2003.

28. Tang Y, Li M, Wang J, Pan Y and Wu FX: CytoNCA: A cytoscape plugin for centrality analysis and evaluation of protein interaction networks. Bio Systems 127: 67-72, 2015.

29. Ogata H, Goto S, Sato K, Fujibuchi W, Bono H and Kanehisa M: KEGG: Kyoto encyclopedia of genes and genomes. Nucleic Acids Res 27: 29-34, 1999.

30. Xie C, Mao X, Huang J, Ding Y, Wu J, Dong S, Kong L, Gao G, Li CY and Wei L: KOBAS 2.0: A web server for annotation and identification of enriched pathways and diseases. Nucleic Acids Res 39: W316-W322, 2011.

31. Cory S and Adams JM: The Bcl2 family: Regulators of the cellular life-or-death switch. Nat Rev Cancer 2: 647-656, 2002.

32. Vaux DL, Cory S and Adams JM: Bcl-2 gene promotes haemopoietic cell survival and cooperates with c-myc to immortalize pre-B cells. Nature 335: 440-442, 1988

33. Fernández-Torres J, Martínez-Nava GA, Gutiérrez-Ruíz MC, Gómez-Quiroz LE and Gutiérrez M: Role of HIF-1 $\alpha$ signaling pathway in osteoarthritis: A systematic review. Rev Bras Reumatol Engl Ed 57: 162-173, 2017 (In Portuguese).

34. Yamashita J, Datta NS, Chun YH, Yang DY, Carey AA, Kreider JM, Goldstein SA and McCauley LK: Role of Bcl2 in osteoclastogenesis and PTH anabolic actions in bone. J Bone Miner Res 23: 621-632, 2008.

35. Cimmino A, Calin GA, Fabbri M, Iorio MV, Ferracin M, Shimizu M, Wojcik SE, Aqeilan RI, Zupo S, Dono M, et al: miR-15 and miR-16 induce apoptosis by targeting BCL2. Proc Natl Acad Sci USA 102: 13944-13949, 2005.

36. Williams LB and Adesida AB: Angiogenic approaches to meniscal healing. Injury 49: 467-472, 2018

37. Zamli Z and Sharif M: Chondrocyte apoptosis: A cause or consequence of osteoarthritis? Int J Rheum Dis 14: 159-166, 2011.

38. Iwata M, Ochi H, Hara Y, Tagawa M, Koga D, Okawa A and Asou Y: Initial responses of articular tissues in a murine high-fat diet-induced osteoarthritis model: Pivotal role of the IPFP as a cytokine fountain. PLoS One 8: e60706, 2013.

39. Chen $\mathrm{H}$ and Tian Y: MiR-15a-5p regulates viability and matrix degradation of human osteoarthritis chondrocytes via targeting VEGFA. Biosci Trends 10: 482-488, 2017.

40. Zelzer E, Mamluk R, Ferrara N, Johnson RS, Schipani E and Olsen BR: VEGFA is necessary for chondrocyte survival during bone development. Development 131: 2161-2171, 2004.
41. Duan X, Murata Y,Liu Y,Nicolae C,Olsen BR and Berendsen AD: Vegfa regulates perichondrial vascularity and osteoblast differentiation in bone development. Development 142: 1984-1991, 2015.

42. Behr B, Tang C, Germann G, Longaker MT and Quarto N: Locally applied VEGFA increases the osteogenic healing capacity of human adipose-derived stem cells by promoting osteogenic and endothelial differentiation. Stem Cells 29: 286-296, 2011.

43. Ruiz Ibán MÁ, Comellas Melero N, Martinez-Botas J, Ortiz A and Diaz Heredia J: Growth factor expression after lesion creation in the avascular zone of the meniscus: A quantitative PCR study in rabbits. Arthroscopy 30: 1131-1138, 2014.

44. Deckers MM, van Bezooijen RL, van der Horst G, Hoogendam J, van Der Bent C, Papapoulos SE and Löwik CW: Bone morphogenetic proteins stimulate angiogenesis through osteoblast-derived vascular endothelial growth factor A. Endocrinology 143: 1545-1553, 2002.

45. Weijts BG, Bakker WJ, Cornelissen PW, Liang KH, Schaftenaar FH, Westendorp B, de Wolf CA, Paciejewska M, Scheele CL, Kent L, et al: E2F7 and E2F8 promote angiogenesis through transcriptional activation of VEGFA in cooperation with HIF1. EMBO J 31: 3871-3884, 2012.

46. Bonkovsky HL: Iron and the liver. Am J Med Sci 301: 32-43, 1991.

47. Carlevaro MF, Albini A, Ribatti D, Gentili C, Benelli R, Cermelli S, Cancedda R and Cancedda FD: Transferrin promotes endothelial cell migration and invasion: Implication in cartilage neovascularization. J Cell Biol 136: 1375-1384, 1997.

48. Senan V, Sucheendran J, Prasad and Balagopal K: Histological features of meniscal injury. Kerala J Orthop 24: 30-36, 2011.

49. Cheng HL, Bryant C, Cook R, O'Connor H, Rooney K and Steinbeck K: The relationship between obesity and hypoferraemia in adults: A systematic review. Obes Rev 13: 150-161, 2012.

50. Semenza G: Signal transduction to hypoxia-inducible factor 1. Biochem Pharmacol 64: 993-998, 2002.

51. Semenza GL: Hypoxia-inducible factor 1: Oxygen homeostasis and disease pathophysiology. Trends Mol Med 7: 345-350, 2001.

52. Zhang FJ, Luo W and Lei GH: Role of HIF- $1 \alpha$ and HIF- $2 \alpha$ in osteoarthritis. Joint Bone Spine 82: 144-147, 2015.

53. Pagès $G$ and Pouysségur J: Transcriptional regulation of the vascular endothelial growth factor gene-a concert of activating factors. Cardiovase Res 65: 564-573, 2005.

54. Liao D and Johnson RS: Hypoxia: A key regulator of angiogenesis in cancer. Cancer Metastasis Rev 26: 281-290, 2007.

55. Rolfs A, Kvietikova I, Gassmann $M$ and Wenger RH: Oxygen-regulated transferrin expression is mediated by hypoxia-inducible factor-1. J Biol Chem 272: 20055-20062, 1997.

56. Trisciuoglio D, Gabellini C, Desideri M, Ragazzoni Y, De Luca T, Ziparo E and Del Bufalo D: Involvement of BH4 domain of bcl-2 in the regulation of HIF-1-mediated VEGF expression in hypoxic tumor cells. Cell Death Differ 18: 1024-1035, 2011.

57. Roos H, Adalberth T, Dahlberg L and Lohmander LS: Osteoarthritis of the knee after injury to the anterior cruciate ligament or meniscus: The influence of time and age. Osteoarthritis Cartilage 3: 261-267, 1995.

This work is licensed under a Creative Commons Attribution-NonCommercial-NoDerivatives 4.0 International (CC BY-NC-ND 4.0) License. 